\title{
Uber Frostblätterigkeit in Lössen und ihre Entstehung
}

\author{
Von Fritz Weidenbach, Stuttgart \\ Mit 4 Abbildungen im Text
}

$\mathrm{Zusammenfassung.} \mathrm{Bei} \mathrm{Frost} \mathrm{entstehen} \mathrm{in} \mathrm{feinkörnigen} \mathrm{Böden} \mathrm{dünne} \mathrm{Eisblätter,}$ durch die bleibende parallele Strukturen erzeugt werden. In Lössen findet man häufig eine fossile Blättrigkeit, die in gleicher Weise durch Frost entstanden ist. Nach rezenten Beobachtungen kann die Aufblätterung in einem einzigen Winter erfolgen. Man sollte daher keine weitgehenden paläoklimatischen Folgerungen aus derartigen Beobachtungen ableiten.

Auch die sog. „Pflugsohle“ der Landwirte (feinblätterige, verdichtete Zone unter dem vom Pflug bearbeiteten Boden) ist als Frostphänomen zu deuten. Sie hat mit der mechanischen $\mathrm{Be}-$ arbeitung des Ackerbodens nichts zu tun.

$\mathrm{Sum} \mathrm{m}$ a r y. A lasting parallel structure in fine grained soil is produced as a result of ice crystals formed through frost. In "Löß" one frequently finds a fossil-like leafiness which originates in the same way - from frost. Following recent observations, this flake-formation can be a result of one winter alone; therefore one should not draw any far-reaching conclusions from such observations.

Also the so-called "Pflugsohle" of the farmers (flaky, condensed zone under ploughed ground) is interpreted as a frost phenomenon. This has nothing to do with the mechanical cultivation of soil.

Viele Lösse sind schichtig. Hier soll jedoch nicht die echte Schichtung in Lössen behandelt werden, wie sie entsteht, wenn feineres und gröberes Material schichtweise miteinander abwechselt. Vielmehr möchte ich auf frostbedingte parallele Strukturen hinweisen, wie sie in Lössen weit verbreitet sind, bisher aber anscheinend nicht genügend beachtet wurden.

Unter Frosteinwirkung wird bei Anwesenheit von Bodenfeuchtigkeit der Löß durch dünne Eisblätter in mehr oder weniger dünne Lagen aufgeblättert. Die einzelnen Lößlagen bleiben nach dem Auftauen der trennenden Eisblätter durch eine Fläche voneinander getrennt, die i. a. wellig verbogen ist. Die Fläche selbst macht den Eindruck, als hätte man sie durch Pressung mit einer Matritze erzeugt. Die Lößblätter sind von sehr verschiedener Dicke. Es gibt Partien mit einer Blattdicke von $1 / 4$ bis $1 / 2 \mathrm{~mm}$, andere haben $1 \mathrm{~cm}$ und mehr.

Es ist bekannt, daß bei Frost das Wasser in lehmigen Böden in einzelnen dünnen Eislammellen gefriert. Man kann im Winter beobachten, wie sich das Eis in ganz regelmäßigen Abständen anordnet, so daß ein gefrorener Boden die Struktur eines Blätterteiges annimmt. Die einzelnen Eisblätter gehen nicht unbegrenzt durch, sie lösen sich vielmehr gegenseitig immer wieder ab. Sie sind auch niemals ganz ebenflächig, sondern immer mindestens leicht gewellt. ${ }^{1}$ )

Nach dem Wegtauen der Eisblätter bleibt die blätterige Parallelstruktur im Boden erhalten. Bezeichnend ist nun, daß diese Blätterigkeit (nicht Schichtigkeit) sowohl in fossiler Form innerhalb des Lösses auftritt wie auch rezent. Man kann in Lehmgruben beobachten, wie jeden Winter aufs Neue durch Frost parallel zur Oberfläche - beispielsweise parallel zu einer Baggerwand - eine blätterige Struktur (oder besser gesagt: Textur) entsteht. Die nachfolgenden Abbildungen der Aufschlüsse und Handstücke zeigen diese Erscheinung deutlich.

Man kann die Grenze der früher gefrorenen Schicht gegen den nicht gefrorenen Boden deutlich erkennen (s. Abb. 1). Bei einer genauen Untersuchung stellt sich heraus,

1) E. SChEnK, 1955, hat in dieser Zeitschrift eine Darstellung über Ursachen und Vorgänge bei der Bildung von Blättereis gegeben. Dort findet sich auf S. 199 auch eine Abbildung, bei der der wellige Verlauf der Eisblätter ganz deutlich zu erkennen ist. 


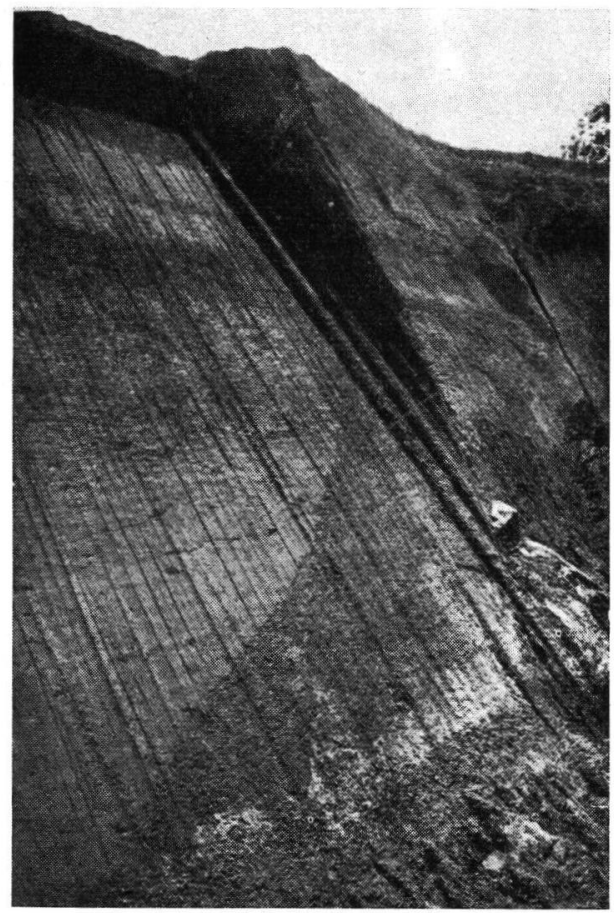

Abb. 1. Lehmgrube Unterweißach, Kreis Backnang. Die Grubenwand ist etwa $6 \mathrm{~m}$ hoch. Rechts die alte Baggerfront von 1954, links die neue von 1955. Der Frost ist im Winter 1953/54 ca. $60 \mathrm{~cm}$ tief senkrecht zur alten Baggerwand eingedrungen. Die Aufblätterungszone ist vom Bagger 1955 unter spitzem Winkel angeschnitten worden. Die Frostgrenze ist in der neuen Baggerwand deutlich zu erkennen, sie verläuft von der linken unteren Ecke zur Mitte des Bildes. Die fossile Frostblättrigkeit in horizontaler Richtung ist ebenfalls erkenntlich. Man beachte, daß nach unten die Blätter immer dicker werden.

daß die einzelnen Lößblätter in der Nähe der Oberfläche sehr dünn sind, nach der Tiefe bzw. ins Innere des Erdreichs aber immer dicker werden. Genau dasselbe beobachten wir nun auch bei fossiler Frostaufblätterung. Auch bei dieser werden die Blätter nach unten immer dicker, sie nehmen nach einigen Dezimetern von der - zwar nicht genau bekannten - früheren Oberfläche entfernt bereits Stärken von mehreren Zentimetern an. In noch größerer Tiefe zeigen sich endlich nur noch in Abständen von Dezimetern im Löß einzelne dünne Trennfugen, die im großen ganzen zwar horizontal, aber doch sehr unregelmäßig verlaufen. Es liegt nahe, auch diese Trennfugen auf Frost zurückzuführen, da sie praktisch nicht von denen innerhalb der dünneren Frostblätter darüber zu unterscheiden bzw. abzutrennen sind.

Die fossilen und die rezenten Erscheinungen sind so täuschend ähnlich, daß man mit Recht annehmen darf, daß beide auf dieselbe Ursache zurückzuführen sind, nämlich auf Frost. Es ist kaum vorstellbar, daß es sich um eine Konvergenzerscheinung handelt, die auf andere Weise entstanden sein könnte.

Es erscheint mir nun von größter Wichtigkeit, daß derartige Strukturen in ihrer genetischen Entstehung richtig gedeutet werden. Mit Schichtung eines Sedimentes hat die Erscheinung nichts zu tun, das wurde bereits oben betont, aber trotzdem sind ganz einwandfrei frostblätterige Lösse als geschichtet bezeichnet worden, und auch ihre Entstehung wurde nicht richtig erkannt. Ich möchte dies an einigen Beispielen näher erörtern. 


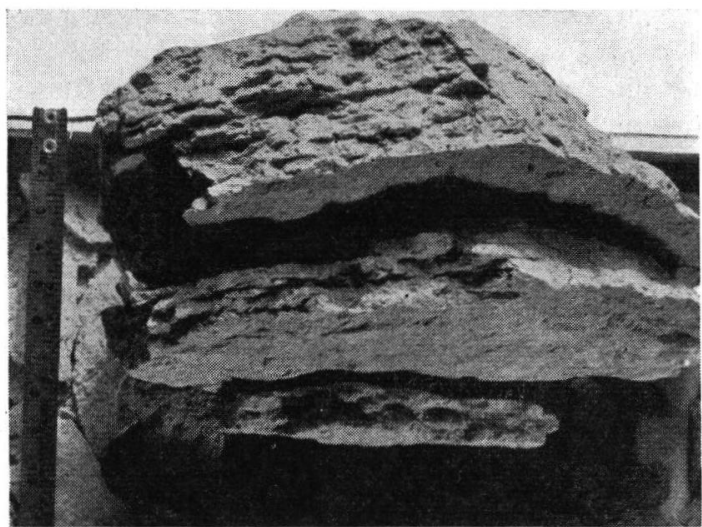

Abb. 2. Lößlehm aus der Lehmgrube Unterweißach, Kreis Backnang init Frostblätterstruktur vom Winter 1953/54. Die Probe ist aus der alten Baggerwand entnommen. Die Blätter liegen parallel zur Baggerwand.

1. In württembergischen Lössen hat H. FreIsING (1951) sogenannte „Naßböden" beschrieben. Dies ist ein großes Verdienst, denn vor ihm wurde nicht erkannt, daß diese Horizonte mit einer gewissen Regelmäßigkeit in weiter Verbreitung auftreten. Diese "Naßböden" haben nun aber - wenigstens zum großen Teil — genau die beschriebene Blätterstruktur. H. Freising hat angenommen, daß die vermeintliche "Schichtigkeit" durch Verschwemmungen und durch Bodenfließen entstanden sei. Aus der Tatsache, daß diese Naßbodenhorizonte im Löß eine Rostfleckigkeit aufweisen, hat er geschlossen, daß feuchtkühles Klima herrschte, wobei er offen läßt, ob zur Zeit ihrer Bildung die Lößaufwehung aufhörte oder fortdauerte.

Diese klimatische Interpretation der Naßböden hatte zur Folge, daß sie als interstadiale Bodenbildungen aufgefaßt wurden, wie dies z. B. durch K. J. N.ARR (1953) geschah (W I/II-Interstadial).

Ich möchte nicht behaupten, daß die „Naßböden“ keine klimatische Ursache haben. Vielleicht hat H. FreIsING vollkommen recht, daß besonders feuchte Witterung z. Zt. ihrer Bildung herrschte. Vielleicht ermöglichte sogar erst eine erhöhte Bodenfeuchtigkeit die Bildung dieser Strukturen; denn zur Entstehung von Blättereis gehört Wasser.

Aber wir haben gesehen, daß eine Aufblätterung nicht längere Zeitperioden braucht, sondern daß schon ein einziger Winter genügt. Es ist also keineswegs angängig, aus einer solchen Erscheinung, wie sie die „Naßböden“ im Löß darstellen, ein Interstadial oder auch nur eine feucht-kühle Periode abzuleiten. Unter einer solchen versteht der Geologe, der Klimatologe, der Geograph usw. immer eine längere Zeit, nicht einen einzigen oder wenige Winter.

Die Rostfleckigkeit der Naßböden als Kriterium für eine feucht-kühle Periode heranzuziehen, ist wohl ebenfalls nicht angängig. Wir haben gesehen, daß durch die Frostaufblätterung eine Parallelstruktur zur Oberfläche erzeugt wird. Dadurch wird natürlich der Wasserstrom in senkechter Richtung gehemmt. Es muß daher im Bereich der Frostblätterigkeit zur Stagnation des Bodenwassers und damit zur Bildung gleyähnlicher Horizonte kommen. Die Gleyfleckigkeit ist also in diesem Falle keine klimatisch bedingte Erscheinung, sondern das Produkt eines geologischen, diagenetischen Vorgangs.

Ich möchte ferner auf eine andere Erscheinung hinweisen, die m. E., was ihre. Entstehungsursache betrifft, ebenfalls einer Überprüfung bedarf. Es handelt sich um die sog. „Pflugsohle“. Von Seiten der Landwirte ist die „B lät ter s truktur “ und Verdichtung in staubkörnigen Böden (Feinlehmen) unter der vom Pflug erreichten Zone 
seit längerer Zeit erkannt. Die blätterigen, verdichteten Böden u n t e r der „Pflugsohle“ (kurz „Pflugsohle“ genannt) wurde nun bisher allgemein darauf zurückgeführt, daß der Pflug mit seiner Schar auf den Böden einen Druck ausübt (E. OstendorfF 1954). „Pflugsohlen" kommen i. a. nur bei Behandlung der Böden mit wenig tiefgehenden Pflügen vor, nicht bei modernen Geräten. Warum? Die modernen Pflïge üben doch einen viel größeren Druck aus!

Nun, die „Pflugsohlen“ haben genau die Struktur der Frostblätterigkeit. Ich kann mir nicht denken, wie ein Pflug überhaupt eine dünnschichtige Anordnung in feinlehmigen Böden zustande bringen kann. Die Erklärung ist viel einfacher. Bei der „Pflugsohle" handelt es sich um den unteren Teil frostaufgeblätterter Feinlehmböden, der u n t e r der Pflugschar erhalten blieb, weil nämlich der Pflug nicht tiefer reichte. Daher findet man die "Pflugsohle“ eben nur auf Äckern, die mit alten, flach wirkenden Pflügen bearbeitet werden.

Vielleicht ist es ein neuer Gesichtspunkt für die Landwirtschaft, daß der Frost nicht nur in günstigem Sinne auf unsere Böden einwirkt, sondern in einem bestimmten $\mathrm{Be}$ reich unter der Oberfläche auch in ungünstigem, wenngleich die Strukturverschlechterung durch Frost - und damit vor allem die ungünstige Veränderung des Wasser-Luft-Haushalts - im wesentlichen auf die Gruppe der schluffigen Feinlehmböden beschränkt ist.

$$
\text { Nachtrag: }
$$

1. Dr. S. Müller, Stuttgart, teilt mir zu obigem Thema nachträglich auf Grund seiner Beobachtungen aus dem Winter 1953 noch mit:

In der humosen, stark belebten Krume mit ihrem Schwammgefüge bilden sich keine blätterigen Eisschichten. Hier hat das Eis ebenfalls Schwammgefüge, z. T. auch nadelige Struktur (Kammeis). Dagegen öffnet der Pflug dem winterlichen Frost den Zugang in den primär weniger belebten und dichter gelagerten Unterboden, der mehr Kapillargefüge hat. Hier entstehen sekundär in schneearmen, kalten Wintern grobe Frostblätter. Primär sind die Blätterstrukturen nicht vorhanden; sonst müßten sie viel häufiger auch an ungepflügten Böden auftreten (Wiesen, Schafweiden, offene Talauen mit lößartigen Böden).

2. Während der Exkursionen im Anschluß an die Tagung der Deutschen Quartärvereinigung in Laufen a. d. Salzach 1955 hatte ich Gelegenheit, in verschiedenen Lößaufschlüssen die beschriebene Frostblättrigkeit in so schöner Ausbildung zu sehen, wie nie zuvor. Ich erwähne hier nur den Aufschluß von St. Georgen, wo im unteren Teil mehrere Meter mächtige, gleyartig veränderte Lösse mit ganz ausgezeichneter Blätterstruktur auftreten. Gerade dieses Vorkommen, das augenscheinlich in einer Geländemulde zur Ablagerung kam, wo der Löß früher wahrscheinlich unter Grundwassereinfluß stand, konnte mich davon überzeugen, daß die Blätterigkeit nur dort - bzw. dann - zur Ausbildung kommt, wo - bzw. wenn - Bodenwasser in ausreichender Menge zur Verfügung steht.

Ich erwähne ferner, daß ich frostblätterige Lösse auch in den so großartigen „Brodelkesseln" auf der Gänserndorfer Terrasse und den höheren Terrassen westlich Seyring sah.

3. Endlich möchte ich darauf hinweisen, daß sich die Blätterstrukturen auch in grobkörnigeren Sedimenten finden, wenn nur die Möglichkeit besteht, daß sich Eisblätter bei Anwesenheit von Wasser bilden können. Ich erwähne hier lediglich, daß ich im Herbst 1955 in weiter, flächenhafter Verbreitung im Stubensandstein Ostwürttembergs (Gegend von Ellwangen) parallel zur Oberfläche verlaufende Strukturen gesehen habe, die zwar wesentlich grobblätteriger sind, als diejenigen im Löß, aber sonst alle Merkmale der echten Frostblätterigkeit aufweisen. Die Strukturen verlaufen quer zur deutlichen Sedimentationsschichtung. 


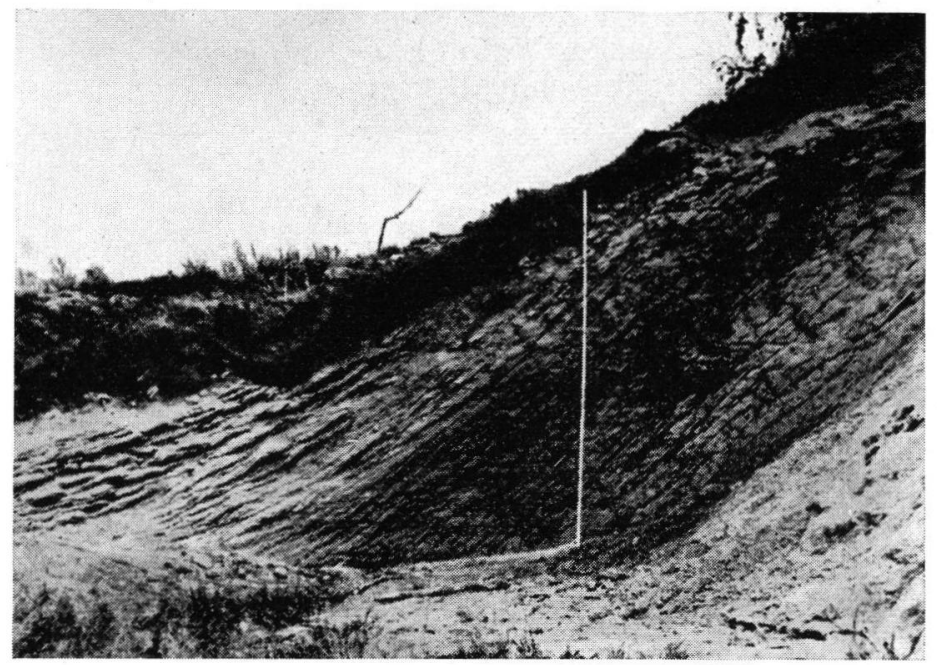

Abb. 3. Sandgrube bei Haselbach, Kreis Ellwangen. Horizontal geschichteter, zersetzter Stubensandstein mit eiszeitlicher Frostblätterung, die etwa paraliel zum Hang verläuft.

Der Stubensandstein ist in dem abgebildeten Aufschluß - wie auch sonst sehr häufig - grobkörnig und sehr mürbe, d. h. wenig verfestigt. Er wird daher auch zu Mauersand abgebaut. Eine gewisse Porosität und damit verbundene Wasseraufnahmefähigkeit ist vorhanden. Sie ist $\mathrm{m}$. E. eine wesentliche Voraussetzung für die Bildung von Eisblättern. Auf den Trennflächen zwischen den einzelnen Blättern befindet sich regelmäßig ein dunkelbraunes Lehm- bzw. Tonhäutchen, das offensichtlich durch Einschwemmung feinster Bodenteilchen von oben her sekundär entstanden ist. Diese Tonhäutchen hemmen natürlich den Wasserdurchstrom in die Tiefe sehr. Ich halte es durchaus für möglich, ja sogar wahrscheinlich, daß diese Erscheinungen eine wesentliche Ursache sind für gewisse Bodenerkrankungen, die gerade auf den ebenen Hochflächen des Stubensandsteins in Ostwürttemberg in den Wäldern sehr häufig auftreten.

Auch hier im Stubensandstein werden die einzelnen Blätter mit der Tiefe immer dicker. Eine Vermessung in der abgebildeten Sandgrube von Haselbach, Kreis Ellwangen, ergab das in Abb. 4 wiedergegebene Diagramm.
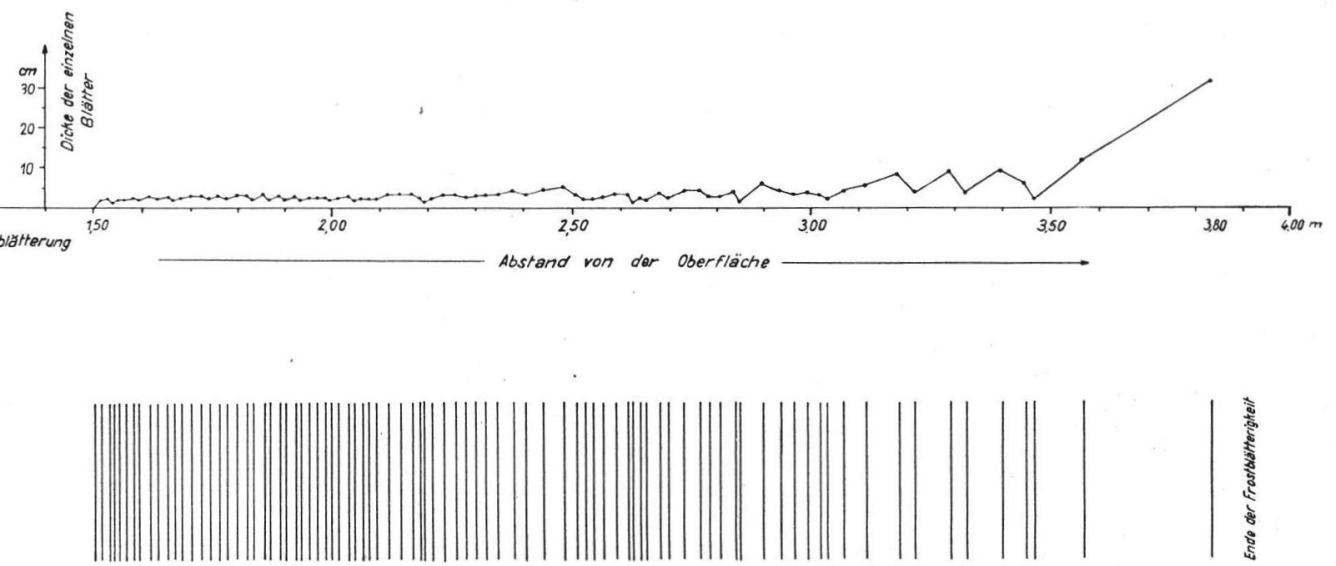

Abb. 4. Diagramm der Frostblätter in der Sandgrube von Haselbach aufgrund einer Vermessung. 
Die ca. 1,50 m mächtige oberste Bodenschicht zeigt keine Blätterstruktur. Die Gesamtmächtigkeit des durchgefrorenen Gesteins beträgt rd. 3,75 m. Offensichtlich handelt es sich daher um eiszeitliche Frostaufblätterung; der Frost geht heute höchstenfalls $1,50 \mathrm{~m}$ tief.

\section{S chriftenverzeichnis:}

Freising, H.: Neue Ergebnisse der Lößforschung im nördlichen Württemberg. - Jh. geol. Abt. württ. statist. L.A. 2, 1951.

NARR, K. J.: Riß oder Würm? - Germania 31, 1953.

OstendorfF, E.: Pflugsohlen. - Aus der Heimat 62, Ohringen 1954.

SCHENK, E.: Die periglazialen Strukturbodenbildungen als Folgen der Hydratationsvorgänge im Boden. - Eiszeitalter und Gegenwart 6, 1955.

Manuskr. eingeg. 28. 1. 1956.

Anschrift d. Verf.: Dr. Fritz Weidenbach, Oberlandesgeologe, Geologisches Landesamt in BadenWürttemberg, Stuttgart, Schützenstraße 4. 